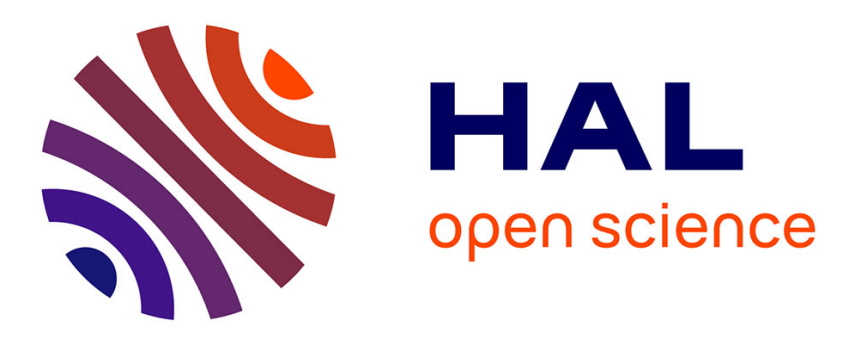

\title{
Nonlocal discrete p-Laplacian Driven Image and Manifold Processing
}

\author{
Abderrahim Elmoataz, Olivier Lezoray, Sébastien Bougleux
}

\section{To cite this version:}

Abderrahim Elmoataz, Olivier Lezoray, Sébastien Bougleux. Nonlocal discrete p-Laplacian Driven Image and Manifold Processing. Comptes rendus de l'Académie des sciences. Série IIb, Mécanique, 2008, 336 (5), pp.428-433. hal-00329514

\section{HAL Id: hal-00329514 https://hal.science/hal-00329514}

Submitted on 12 Oct 2008

HAL is a multi-disciplinary open access archive for the deposit and dissemination of scientific research documents, whether they are published or not. The documents may come from teaching and research institutions in France or abroad, or from public or private research centers.
L'archive ouverte pluridisciplinaire HAL, est destinée au dépôt et à la diffusion de documents scientifiques de niveau recherche, publiés ou non, émanant des établissements d'enseignement et de recherche français ou étrangers, des laboratoires publics ou privés. 


\title{
Nonlocal discrete p-Laplacian Driven Image and Manifold Processing ${ }^{1}$
}

\author{
Abderrahim Elmoataz $^{\mathrm{a}}$, Olivier Lezoray ${ }^{\mathrm{a}, 2}$ Sébastien Bougleux ${ }^{\mathrm{a}}$ \\ ${ }^{a}$ Université de Caen Basse-Normandie and ENSICAEN, GREYC UMR CNRS 6072, 6 Bd. Maréchal Juin, F-14050 \\ CAEN, France \\ Received $* * * * *$; accepted after revision +++++ \\ Presented by
}

\begin{abstract}
A framework for non local discrete p-Laplacian regularization on Image and Manifold represented by weighted graphs of the arbitrary topologies is proposed. The proposed discrete framework unifies the local and non local regularization for image processing and extends them to the processing of any discrete data living on graphs. To cite this article: A. Elmoataz, O. Lezoray, S. Bougleux, C. R. Mecanique XXX (200X).
\end{abstract}

\section{Résumé}

Traitement d'images et de données basé sur le p-Laplacian discret non local. Un cadre genéral pour la régularisation basée sur le p-Laplacien discret pour le traitement d'images et de données représentés par des graphes pondérés de topologies arbitraires est proposé dans cet article. Ce cadre unifie la régularisation locale ou non locale sur les images et l'étend naturellement au traitement de données discrètes sur graphes. Pour citer cet article : A. Elmoataz, O. Lezoray, S. Bougleux, C. R. Mecanique XXX (200X).

Key words: Computer science; Nonlocal discrete regularization; Weighted graph ; p-Laplacian

Mots-clés : Informatique, algorithmique; Régularisation discrète non locale; Graphe pondéré; p-Laplacien

Email addresses: abder.elmoataz@greyc.ensicaen.fr (Abderrahim Elmoataz), olivier.lezoray@unicaen.fr (Olivier Lezoray), sebastien.bougleux@greyc.ensicaen.fr (Sébastien Bougleux).

1 This research work was partially supported by the ANR foundation under grant ANR-06-MDCA-008-01/FOGRIMMI.

2 Corresponding author. 


\section{Version française abrégée}

La régularisation d'images par méthodes variationelles a été utilisée avec succès pour résoudre un grand nombre d'applications en traitement et analyse d'images [1]. Cependant, les fonctionnelles de régularisation usuelles utilisent des opérateurs différentiels qui sont par nature locaux. Depuis les travaux de Buades et Morel [2], sur le filtrage d'image par moyennes non locales, plusieurs travaux récents ont montré l'intérêt d'introduire des fonctionnelles de régularisation non locales pour prendre en compte des interactions plus complexes et introduire plus de flexibilité dans les fonctionnelles de régularisation. Kindermann, Osher et Jones [3] ont été les premiers à interpréter le filtre à moyennes non locales ainsi que d'autres filtres de voisinage sous forme d'une régularisation d'image basée sur des fonctionnelles non locales. Récemment, Gilboa et Osher [4] ont proposé une fonctionnelle quadratique non locale pour la régularisation d'image et la segmentation semi-supervisée qui se veut plus efficace et plus flexible que les méthodes de régularisation locales. Ces travaux peuvent être considérés comme des analogues non locaux des modèles de régularisation basés sur la variation totale. Cependant, ces méthodes ont été développées en supposant que les images sont continues dans un domaine continu, et la discrétisation des équations aux dérivées partielles utilisées pour les résoudre n'est pas sans poser de problèmes lorsque les images sont définies sur des domaines irréguliers ou sur des espaces de grandes dimensions. Dans cet article, nous proposons un cadre genéral pour la régularisation basée sur le p-Laplacien discret pour le traitement d'images et de données représentés par des graphes pondérés de topologies arbitraires. Ce cadre unifie la régularisation locale ou non locale sur les images et l'étend naturellement au traitement de données discrètes sur graphes. Toutes les méthodes de régularisation continues locales ou non locales avec une discrétisation donnée peuvent s'interpréter comme des cas particuliers de notre cadre général discret. Comme dans ce cadre la régularisation est directement exprimée en discret, cela ne nécéssite aucune résolution d'équations aux dérivées partielles. Nous considérons une image, ou toute fonction définie sur un domaine discret, comme une fonction définie sur un graphe pondéré $G_{w}=(V, E)$ où $V$ est un ensemble de sommets, $E \subset V \times V$ un ensemble d'arêtes et $w$ une fonction de similarité définie sur l'ensemble des arêtes. La régularisation discrète non locale de $f^{0} \in \mathcal{H}(V)$ est définie par la minimisation suivante :

$$
\min _{f \in \mathcal{H}(V)}\left\{E_{w}^{p}\left(f, f^{0}, \lambda\right)=\frac{1}{p} \sum_{v \in V}\left\|\nabla_{w} f\right\|^{p}+\frac{\lambda}{2}\left\|f-f^{0}\right\|_{\mathcal{H}(V)}^{2}\right\},
$$

où $\mathcal{H}(V)$ est un espace de Hilbert défini sur les sommets de $G_{w}, p \in[1,+\infty)$ est le dégré de régularité, $\lambda$ est un paramètre d'attache aux données et $\nabla_{w} f$ représente le gradient pondéré d'une fonction $f$ sur le graphe. Nous introduisons alors des opérateurs différentiels sur graphe dont le gradient pondéré et le p-Laplacien (équations (3) et (5)). Puis, nous montrons que la minimisation de (1) peut se traduire comme l'analogue discret des équations d'Euler-Lagrange et la solution peut être obtenue par un algorithme de type Gauss-Jacobi (équation (10)) ; ceci amène à des processus de filtrage simples et rapides paramétrés par la fonction de pondération $w$ et le degré de régularité $p$. Ces équations peuvent s'appliquer de la même manière sur des images, des maillages ou des données de dimensions quelconques en adaptant simplement la topologie du graphe et la fonction de pondération (Figures 1 et 2).

\section{Introduction}

Regularization by variational methods has shown its effectiveness for many applications. However, there are some limitations in the functionals used in regularization such as total variational models or active contour models (see [1] and references therein). Indeed, the latter are based on derivatives which only consider local features of the data. Since the advent of the non local means filter [2], the use of non local 
interactions, to capture the complex structures of the data, has received a lot of attention and has shown to be very effective and allows much more flexibility in the regularization process. Kindermann, Osher and Jones [3] were the first to interpret non local means and neighborhood filters as regularization based on non local functionals. Later, Gilboa and Osher [4] have proposed a non local quadratic functional of weighted differences for image regularization and semi-supervised segmentation. These works can be considered as the non local analogues of Total Variation models for image regularization. Most of the proposed regularization processes have been proposed in the context of image processing where images are considered as continuous functions on continuous domains. Then, one considers a continuous energy functional which is classically solved by the corresponding Euler-Lagrange equation or its associated flow. However, the discretization of the underlying differential operators is difficult for high dimensional data and for image and data defined on irregular domains.

In this paper, we propose a framework for non local discrete p-Laplacian regularization on Image and Manifold represented by weighted graphs of the arbitrary topologies [5]. The proposed discrete framework unifies local and non local regularization for image processing and extends them to the processing of any discrete data living on graphs. All the continuous regularization methods (local or non local) with a given discretization scheme can be considered as particular cases of our proposed discrete regularization. Since the proposed framework is directly expressed in a discrete setting, no partial difference equations resolution is needed. The proposed regularization enables local or nonlocal regularization by using appropriated graphs topologies and edge weights. Let $G_{w}=(V, E)$ be a weighted graph consisting in a set of vertices $V$, a set of edges $E \subset V \times V$, and a similarity weight function $w$ defined on edges. Let $\mathcal{H}(V)$ be a Hilbert space defined on the vertices of $G_{w}$. We formalize the discrete data regularization of a function $f^{0} \in \mathcal{H}(V)$ by the following minimization problem:

$$
\min _{f \in \mathcal{H}(V)}\left\{E_{w}^{p}\left(f, f^{0}, \lambda\right)=\frac{1}{p} \sum_{v \in V}\left\|\nabla_{w} f\right\|^{p}+\frac{\lambda}{2}\left\|f-f^{0}\right\|_{\mathcal{H}(V)}^{2}\right\},
$$

where $p \in[1,+\infty)$ is the smoothness degree, $\lambda$ is the fidelity parameter, and $\nabla_{w} f$ represents the weighted gradient of the function $f$ over the graph. The solution of problem (2) leads to a family of nonlinear processing methods, parameterized by the weight function, the degree of smoothness, and the fidelity parameter.

\section{Discrete p-Laplacian regularization}

In this section, we recall some basic definitions on graphs and we introduce discrete differential operators [6] and the p-Laplacian on graphs which can be considered as a discrete analogue of the continuous pLaplacian.

\subsection{Preliminary definitions}

A graph $G_{w}=(V, E)$ consists in a finite set $V$ of $N$ vertices and a finite set $E \subseteq V \times V$ of edges. We assume $G_{w}$ to be undirected, with no self-loops and no multiple edges. Let $(u, v)$ be the edge that connects the vertices $u$ and $v$. An undirected graph is weighted if it is associated with a weight function $w: E \rightarrow \mathbb{R}_{+}$satisfying $w(u, v)=w(v, u)$, for all $(u, v) \in E$, and $w(u, v)=0$ if $(u, v) \notin E$. The weight function represents a similarity measure between two vertices of the graph. We use the notation $u \sim v$ for two adjacent vertices. Let $\mathcal{H}(V)$ denote the Hilbert space of real-valued functions on vertices. A function $f: V \rightarrow \mathbb{R}^{m}$ in $\mathcal{H}(V)$ assigns a vector $f(v)$ to each vertex $v \in V$. Clearly, $f$ can be represented by 
a column vector of $\mathbb{R}^{N}, f=\left[f_{1}, \ldots, f_{N}\right]^{T}$. By analogy with functional space we define $\int_{V} f=\sum_{V} f(u)$. The function space $\mathcal{H}(V)$ is endowed with the usual inner product $\langle f, h\rangle_{\mathcal{H}(V)}:=\sum_{v \in V} f(v) h(v)$, where $f, h \in \mathcal{H}(V)$. Similarly, one can define $\mathcal{H}(E)$, the space of real-valued functions on edges.

\subsection{Weighted Gradient and Divergence Operators}

Let $G_{w}=(V, E)$ denote a weighted graph. The difference operator $d: \mathcal{H}(V) \rightarrow \mathcal{H}(E)$ of a function $f \in \mathcal{H}(V)$ on an edge $(u, v) \in E$, is defined by: $(d f)(u, v):=\sqrt{w(u, v)}(f(v)-f(u)), \forall(u, v) \in E$. The directional derivative (or edge derivative) of a function $f \in \mathcal{H}(V)$ at a vertex $v$ along an edge $e=(u, v)$, is defined as $\partial_{v} f_{u}:=(d f)(u, v)$. The weighted gradient operator $\nabla_{w}$ of a function $f \in \mathcal{H}(V)$ at a vertex $v$ is the vector operator defined by $\nabla_{w} f(v)=\left(\partial_{u} f(v): u \sim v\right)^{T}$. The local variation of $f$ at $v$, is defined to be:

$$
\left\|\nabla_{w} f(v)\right\|:=\sqrt{\sum_{u \sim v}\left(\partial_{u} f(v)\right)^{2}}=\sqrt{\sum_{u \sim v} w(u, v)(f(u)-f(v))^{2}} .
$$

It can be viewed as a measure of the regularity of a function around a vertex. The adjoint operator of the difference operator, denoted by $d^{*}: \mathcal{H}(E) \rightarrow \mathcal{H}(V)$, is defined by $\langle d f, h\rangle_{\mathcal{H}(E)}:=\left\langle f, d^{*} h\right\rangle_{\mathcal{H}(V)}$, with $f \in \mathcal{H}(V)$ and $h \in \mathcal{H}(E)$. Using the definitions of the inner products in $\mathcal{H}(V)$ and $\mathcal{H}(E)$, and of the difference operator, we obtain the expression of $d^{*}$ at a vertex $v$ :

$$
\left(d^{*} h\right)(v)=\sum_{u \sim v} \sqrt{w(u, v)}(h(u, v)-h(v, u)) .
$$

The divergence operator, defined by $-d^{*}$, measures the net outflow of a function in $\mathcal{H}(E)$ at each vertex of $V$.

\subsection{A Family of Weighted p-Laplace Operators}

The weighted $p$-Laplace operator, $\Delta_{w}^{p}: \mathcal{H}(V) \rightarrow \mathcal{H}(V)$ with $1 \leq p<+\infty$, is defined by $\Delta_{w}^{p} f:=$ $\frac{1}{p} d^{*}\left(\left\|\nabla_{w} f\right\|^{p-2} d f\right)$. Substituting the difference operator and (4) into the definition of $\Delta_{w}^{p} f$, we obtain the expression of $\Delta_{w}^{p}$ at a vertex $v$ :

$$
\Delta_{w}^{p} f(v)=\frac{1}{p} \sum_{u \sim v} \gamma(u, v)(f(v)-f(u)),
$$

where $\gamma$ is the function defined by $\gamma(u, v):=w_{u v}\left(\left\|\nabla_{w} f(v)\right\|^{p-2}+\left\|\nabla_{w} f(u)\right\|^{p-2}\right)$. The operator $\Delta_{w}^{p}$ is nonlinear, with the exception of $p=2$. Furthermore, $\Delta_{w}^{p}$ is positive semi-definite:

$$
\begin{aligned}
\left\langle f, p \Delta_{w}^{p} f\right\rangle_{\mathcal{H}(V)} & =\left\langle f, d^{*}\left(\left\|\nabla_{w} f\right\|^{p-2} d f\right)\right\rangle_{\mathcal{H}(V)}=\left\langle d f,\left\|\nabla_{w} f\right\|^{p-2} d f\right\rangle_{\mathcal{H}(E)} \\
& =\sum_{v \in V}\left\|\nabla_{w} f(v)\right\|^{p-2} \sum_{u \sim v}((d f)(u, v))^{2}=\sum_{v \in V}\left\|\nabla_{w} f(v)\right\|^{p} \geq 0 .
\end{aligned}
$$

The definition of $\Delta_{w}^{p}$ can be considered as the discrete analogue of the $p$-Laplacian in the continuous case. When $p=2, \Delta_{w}^{2}$ represents the weighted Laplace operator on $G_{w}$, and (5) reduces to: $\Delta f(v):=$ $\Delta_{w}^{2} f(v)=\sum_{u \sim v} w(u, v)(f(v)-f(u))$. When $p=1, \Delta_{w}^{1}$ represents the weighted curvature operator on $G_{w}$, and expression (5) reduces to: $\kappa f(v):=\Delta_{w}^{1} f(v)=\frac{1}{2} \sum_{u \sim v} w(u, v)\left(\frac{1}{\left\|\nabla_{w} f(v)\right\|}+\frac{1}{\left\|\nabla_{w} f(u)\right\|}\right)(f(v)-f(u))$. In practice, to avoid zero denominator in the curvature computation, the local variation (3) is replaced by its regularized version: $\left\|\nabla_{w} f(v)\right\|_{\epsilon}:=\sqrt{\left\|\nabla_{w} f(v)\right\|^{2}+\epsilon^{2}}$, with $\epsilon \rightarrow 0$ a fixed small constant. 


\section{3. p-Laplace Regularization on Weighted Graphs}

In this Section, one considers a general function $f^{0}: V \subset \mathbb{R}^{n} \rightarrow \mathbb{R}^{m}$ defined on graphs of the arbitrary topologies and we want to regularize this function. In a given context, the function $f^{0}$ represents an observation of a clean function $g: V \rightarrow \mathbb{R}$ corrupted by a given noise $n$ such that $f^{0}=g+n$. Such noise is assumed to have zero mean and variance $\sigma^{2}$, which usually corresponds to observation errors. The regularization of such a function corresponds to an optimization problem which can be formalized by the minimization of a weighted sum of two energy terms:

$$
f^{*}=\min _{f \in \mathcal{H}(V)}\left\{E_{w}^{p}\left(f, f^{0}, \lambda\right):=\frac{1}{p} \sum_{v \in V}\left\|\nabla_{w} f(v)\right\|^{p}+\frac{\lambda}{2}\left\|f-f^{0}\right\|_{\mathcal{H}(V)}^{2}\right\} .
$$

The first term in (7) is the smoothness term or regularizer, meanwhile the second is the fitting term. The parameter $\lambda \geq 0$ is a fidelity parameter, called the Lagrange multiplier, which specifies the trade-off between the two competing terms. Both terms of the energy $E_{w}^{p}$ are strictly convex functions of $f$. In particular, by standard arguments in convex analysis, the problem (7) has a unique solution, for $p=1$ and $p=2$, which satisfies:

$$
\left.\frac{\partial E_{w}^{p}\left(f, f^{0}, \lambda\right)}{\partial f}\right|_{v}=\frac{1}{p} \frac{\partial}{\partial f}\left\|\nabla_{w} f(v)\right\|^{p}+\lambda\left(f(v)-f^{0}(v)\right)=\Delta_{w}^{p} f(v)+\lambda\left(f(v)-f^{0}(v)\right)=0, \quad \forall v \in V .
$$

The solution of problem (7) is also the solution of the system of equations (8). This is a nonlinear system, with the exception of $p=2$. Substituting the expression of the $p$-Laplace operator into (8), we obtain:

$$
\left(\lambda+\frac{1}{p} \sum_{u \sim v} \gamma(u, v)\right) f(v)-\frac{1}{p} \sum_{u \sim v} \gamma(u, v) f(u)=\lambda f^{0}(v), \quad \forall v \in V .
$$

Among the existing methods to solve the system of equations (9), we use the Gauss-Jacobi iterative algorithm. Let $t$ be an iteration step, and let $f^{(t)}$ be the solution of equation (9) at the step $t$. The corresponding linearized Gauss-Jacobi algorithm is given by:

$$
\left\{\begin{array}{l}
f^{(0)}=f^{0} \\
\gamma^{(t)}(u, v)=w(u, v)\left(\left\|\nabla_{w} f^{(t)}(v)\right\|^{p-2}+\left\|\nabla_{w} f^{(t)}(u)\right\|^{p-2}\right), \quad \forall(u, v) \in E \\
f^{(t+1)}(v)=\left(p \lambda+\sum_{u \sim v} \gamma^{(t)}(u, v)\right)^{-1}\left(p \lambda f^{0}(v)+\sum_{u \sim v} \gamma^{(t)}(u, v) f^{(t)}(u)\right), \quad \forall v \in V
\end{array}\right.
$$

where $\gamma^{(t)}$ is the function $\gamma$ at the step $t$. The weights $w(u, v)$ are computed from $f^{0}$, or can be given a priori. We define the function $\varphi$ at an iteration $t$ of algorithm (10) by:

$$
\varphi^{(t)}(v, u)=\frac{\gamma^{(t)}(u, v)}{p \lambda+\sum_{u \sim v} \gamma^{(t)}(u, v)} \text { if } u \neq v, \text { and } \varphi^{(t)}(v, v)=\frac{p \lambda}{p \lambda+\sum_{u \sim v} \gamma^{(t)}(u, v)}
$$

Then, an iteration of the regularization algorithm (10) is rewritten as:

$$
f^{(t+1)}(v)=\varphi^{(t)}(v, v) f^{0}(v)+\sum_{u \sim v} \varphi^{(t)}(v, u) f^{(t)}(u), \forall v \in V .
$$

At each iteration, the new value $f^{(t+1)}$, at a vertex $v$, depends on two quantities, the original value $f^{0}(v)$, and a weighted average of the existing values in a neighborhood of $v$. This shows that the proposed filter, obtained by iterating (11), is a low-pass filter which can be adapted to many graph structures and weight functions. 


\section{Examples}

In this Section, we present some examples of the proposed method on Images (Figure 1(a)-(e)), Meshes (Figure 1(f)-(h)) and Data base images (i.e. Manifolds, see Figure 2). A grid graph is considered for image processing $\left(f^{0}: V \subset \mathbb{R}^{2} \rightarrow \mathbb{R}^{3}\right)$, a neighborhood graph is considered for mesh processing $\left(f^{0}: V \subset \mathbb{R}^{3} \rightarrow\right.$ $\left.\mathbb{R}^{3}\right)$ and a complete graph is considered for data processing $\left(f^{0}: V \rightarrow \mathbb{R}^{16 \times 16}\right)$.

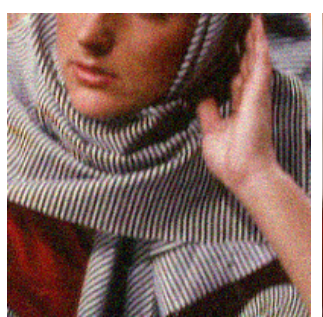

(a) Noisy image $(\sigma=(\mathrm{b}) p=2$, local process$15)$. g.

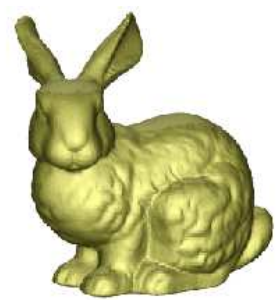

(f) Original Bunny

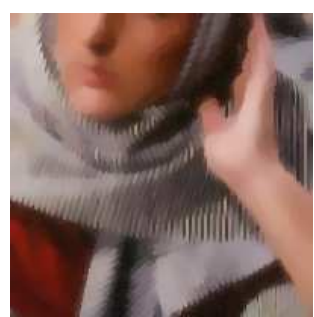

(c) $p=1$, local processing.

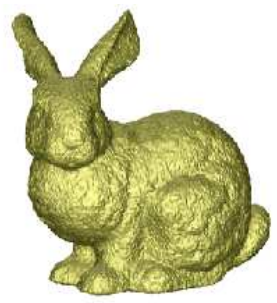

(g) Noisy Bunny
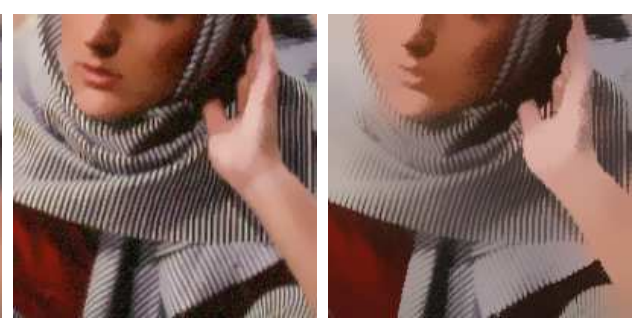

cessing $(3 \times 3$ patch $)$.

(e) $p=1$, nonlocal pro-

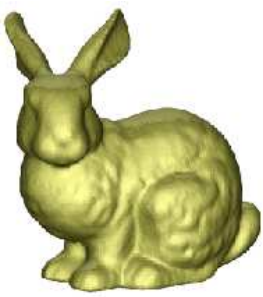

(h) Regularized $(p=2)$

Figure 1. Processing examples for different values of $p$ for local and nonlocal image and mesh processing.

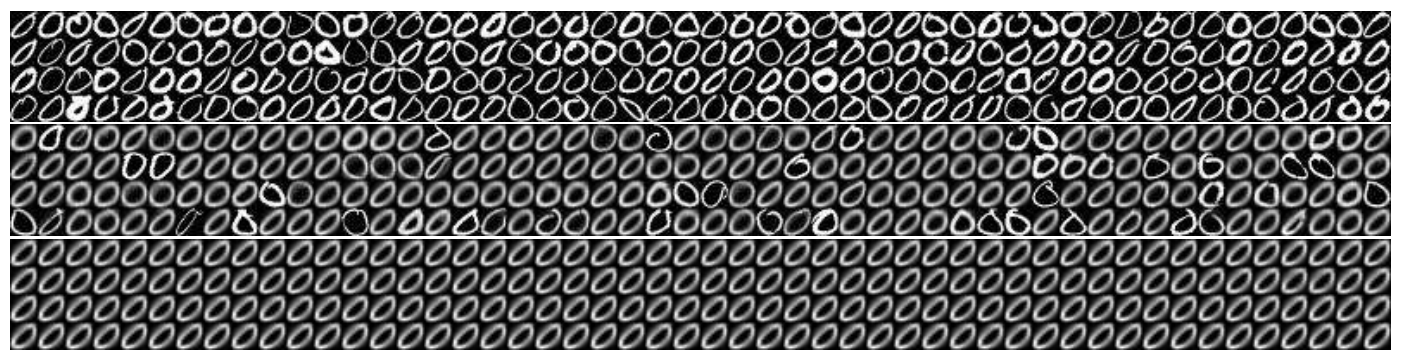

Figure 2. Nonlocal manifold regularization on USPS handwritten digit 0 database (first line). Second line: regularization with $\lambda=0.01$. Third line: regularization with $\lambda=0$.

\section{References}

[1] T.F. Chan, J. Shen. Image Processing and Analysis - variational, PDE, wavelets, and stochastic methods. SIAM, 2005.

[2] A. Buades, B. Coll, J-M. Morel. A review of image denoising algorithms, with a new one. Multiscale Modeling and Simulation, 4(2)(2005) 490-530.

[3] S. Kindermann, S Osher, P.W. Jones. Deblurring and denoising of images by nonlocal functionales. Multiscale Modeling and Simulation, 4(4)(2005) 1091-1115. 
[4] G. Gilboa, S. Osher. Nonlocal linear image regularization and supervised segmentation. Multiscale Modeling and Simulation (MMS), 6(2)(2007) 595-630.

[5] O. Lezoray, A. Elmoataz, S. Bougleux. Graph regularization for color image processing. Computer Vision and Image Understanding (CVIU), 107(1-2)(2007) 38-55.

[6] A. Bensoussan and J-L. Menaldi. Difference equations on weighted graphs. Journal of Convex Analysis, 12(1)(2005) 13-44. 